\title{
Deformación de la Aleta Caudal en Alevín de Salmón del Atlántico (Salmo salar), en Etapa de Saco Vitelino
}

\author{
Deformation of the Caudal Fin in Atlantic Salmon fry (Salmo salar), in the Yolk Sac Stage
}

\author{
Mariana Rojas ${ }^{1}$; Mario Pellón ${ }^{2}$; Claudio Cerda ${ }^{3}$; Roberto Ulloa ${ }^{4}$; Rudy Suarez ${ }^{5}$; Marcos Godoy ${ }^{5,6,7} \&$ Mariano del Sol $^{8}$
}

ROJAS, M.; PELLÓN, M.; CERDA, C.; ULLOA, R.; SUAREZ, R.; GODOY, M. \& DEL SOL, M. Deformación de la aleta caudal en alevín de Salmón del Atlántico (Salmo salar), en etapa de saco vitelino. Int. J. Morphol., 38(6):1631-1638, 2020.

RESUMEN: En este estudio se analiza una deformación que afectó la aleta caudal de los alevines de salmón del Atlántico (Salmo salar) y que les proporcionó un aspecto de "cola aguzada". Al momento de la eclosión se observaron completamente normales pero la deformación se detectó con posterioridad. El objetivo de este trabajo es describir los cambios anatómicos e histológicos de la aleta caudal deformada del alevín de salmón del Atlántico (Salmo salar). Para esto se utilizaron 60 alevines para realizar análisis de laboratorio y descartar la presencia de patógenos virales o bacterianos. Otros 60 alevines con un estado de desarrollo entre 600 y 700 Unidades térmicas acumuladas (UTAs) fueron anestesiados con Benzocaína $5 \%$, fijados en formalina al $10 \%$ pesados y medidos. De estos un grupo de 30 alevines (15 normales y 15 deformes) fueron sometidos a la técnica de Hanken y Wassersug para evaluar Lepidotriquias. Los otros 30 alevines (15 normales y 15 deformes), fueron procesados mediante las técnicas histoquímicas: H\&E/azul de Alcián para evaluar las características histológicas generales. Adicionalmente se utilizó técnicas inmunohistoquímicas para reconocer la ubicación y la presencia de los centros de señales Sonic hedgehog (Shh) para la formación de Lepidotriquias. A los valores obtenidos para las variables cuantitativas peso y longitud de cuerpo, largo y ancho de aleta caudal, se les realizó estadística descriptiva y fueron sometidos a prueba de normalidad de Shapiro-Wilk. Las diferencias observadas entre peces normales y deformes, fueron analizadas mediante prueba t de Student o U de Mann Whitney, utilizando el paquete estadístico IBM SPSS 20.0. La deformación se observó desde las 600 UTA. El peso de los alevines deformes fue similar al de los peces normales ( $p>0,05)$, lo mismo sucedió con la longitud de la aleta ( $p>0,05)$. Por el contrario, el ancho de la aleta de los deformes fue muy reducida $(\mathrm{p}<0,05)$. El fenotipo de aleta aguzada presentó un cambio en la integridad de los bordes, fracturas de Lepidotriquias. La epidermis de la aleta caudal de los alevines deformados presentó sus centros de señalización Shh activos, pero el blastema interrayos presentó vasodilatación, congestión y hemorragias. La presentación de este caso se relacionó con incrementos bruscos de temperatura peri eclosional.

PALABRAS CLAVE: Salmón del Atlántico, Salmo salar; deformación, aleta caudal.

\section{INTRODUCCIÓN}

Quint et al. (2002) describieron que el patrón óseo en la aleta caudal puede ser alterado por la expresión ectópica de morfógenos tales como Sonic Hedgehog (Shh) y proteína morfogenética del hueso (BMP2b).

En 2015 Rojas et al. realizaron la descripción de la aleta caudal normal del alevín de Salmón del Atlántico $(S$. salar). En el presente se realizó un análisis comparativo de los cambios anatómicos e histológicos de la aleta caudal del alevín post-eclosional deformada y se evaluó la expresión del morfógeno Shh durante un episodio accidental que estuvo relacionado con cambios de la temperatura.

Como hasta el momento no se ha reportado las características morfológicas de la aleta aguzada en estadios post eclosionales tan tempranos, en este estudio se presenta una deformación de la aleta caudal que afectó a los alevines con saco vitelino de Salmón del Atlántico (Salmo salar) en el Sur de Chile, durante el año 2012, caracterizada por presentación de la aleta caudal con aspecto de "cola aguzada".

\footnotetext{
${ }^{1}$ Laboratorio Embriología Comparada. Programa de Anatomía y Biología del Desarrollo, Facultad de Medicina, ICBM. Universidad de Chile, Chile.

${ }^{2}$ Facultad de Medicina. Universidad de La Frontera, Temuco, Chile.

${ }^{3}$ Universidad San Sebastián, Facultad de Medicina Veterinaria, Sede De la Patagonia, Lago Panguipulli 1390, Puerto Montt, 5480000, Chile.

${ }^{4}$ Piscicultura Hornopirén, Friosur, Hornopiren, Chile.

${ }^{5}$ Centro de Investigaciones Biológicas Aplicadas (CIBA), Puerto Montt, 5480000, Chile.

${ }^{6}$ Laboratorio de Biotecnología Aplicada, Facultad de Medicina Veterinaria, Universidad San Sebastián, Sede De La Patagonia, Lago Panguipulli 1390, Puerto Montt, 5480000, Chile.

${ }^{7}$ Doctorado en Acuicultura, Programa Cooperativo Universidad de Chile, Universidad Católica del Norte,Pontificia Universidad Católica de Valparaíso, Coquimbo 17811421, Chile.

${ }^{8}$ Centro de Excelencia en Estudios Morfológicos y Quirúrgicos (CEMyQ), Universidad de La Frontera, Chile.
} 


\section{MATERIAL Y MÉTODO}

Las ovas fueron obtenidas de reproductores que realizaron su ciclo de vida completa en agua dulce, las cuales se caracterizan genéticamente por presentar un locus de rasgo cuantitativo (QTL) para la resistencia a la necrosis pancreática infecciosa (IPN).

Las ovas $(\mathrm{N}=3.661 .281)$ fueron incubadas en piscicultura primaria a temperaturas que variaron entre los 5,3 a $8^{\circ} \mathrm{C}$ (temperatura de río) hasta las 306 y 319 Unidades térmicas acumuladas (UTAs). Posteriormente fueron trasladadas a una piscicultura definitiva, siendo la temperatura de ingreso de $3{ }^{\circ} \mathrm{C}$, luego se llevó a cabo, un proceso de aclimatación, que consistió en incrementar la temperatura de cultivo de forma gradual hasta llegar a $\operatorname{los} 5{ }^{\circ} \mathrm{C}$. Repentinamente, el sistema presentó un aumento de la temperatura hasta los 10 ${ }^{\circ} \mathrm{C}$. Transcurrido 5 días de iniciado el evento se redujo la temperatura a $6{ }^{\circ} \mathrm{C}$ (a razón de un grado por día). Después de 25 días se realizó una aclimatación previa al traslado a la fase de cultivo de primera alimentación, subiéndose la temperatura de cultivo gradualmente a $10{ }^{\circ} \mathrm{C}$. Durante el cultivo la saturación de oxígeno fue de $100 \%$ (normoxia).

Para la descripción morfológica macroscópica se realizó un análisis de 638 peces, pertenecientes a tres lotes, mantenidos en 3 unidades de cultivo diferentes, utilizando una lupa esteroscópica (Leica). Los hallazgos en la aleta caudal fueron clasificados como normales (sin hallazgos), leves a moderados (se observaba una alteración de la forma de la aleta caudal) y severo (se observaba un plegamiento de los rayos de la aleta caudal, dando la apariencia de una aleta aguzada).

Con el fin de conocer el estado sanitario de los peces, se realizó análisis de laboratorio de 60 especímenes, para determinar la condición sanitaria respecto del Virus de la necrosis pancreática infecciosa (IPNV), Virus de la anemia infecciosa del salmón (ISAV), Piscine orthoreovirus (PRV1,2 y 3), Flavobacterium psychrophilum y Renibacterium salmoninarum de acuerdo a las condiciones para la reacción en cadena de la polimerasa (PCR), secuencias de partidores y sondas previamente descritas (Blake et al., 1995; Wiklund et al., 2000; Chase et al., 2006; Snow et al., 2006; Olsen et al., 2015; Takano et al., 2016). Adicionalmente muestras de branquias y riñón fueron inoculadas en agar tripticasa soya (Whitman, 2004) y Anacker \& Ordal (1959) e incubadas a 22 ${ }^{\circ} \mathrm{C}$ y $15{ }^{\circ} \mathrm{C}$, respectivamente para la determinación de patógenos bacterianos.

Adicionalmente se procedió a analizar otros 60 alevines que tenían entre 600 y 700 UTAs, los cuales fueron pesados y posteriormente sacrificados con Benzocaína al $5 \%$ (BZ-20®,
Veterquímica) disuelta en el agua a una dosis de $800 \mathrm{mg} / \mathrm{l}$, (de acuerdo a normas éticas de investigación con animales), luego fueron fijados en formalina tamponada al $10 \%$, por 48 horas. Previo a su procesamiento se evaluaron las características morfológicas de la aleta caudal tales como longitud y ancho.

Para la descripción morfológica mesoscópica se utilizaron 30 alevines, los que fueron sometidos a digestión proteica (tripsina) y expuestos al colorante azul de Alcián, que tiñe de color azul a los glicosaminoglicanos de las Lepidotriquias (Rojas et al., 2015). Los ejemplares diafanizados fueron observados y fotografiados con un microscopio Zeiss Axiostar plus que tiene polarizadores adaptados.

Para la descripción histológica se utilizaron 30 alevines los que fueron incluidos en paraplast, seccionados en cortes sagitales seriados de $5 \mu \mathrm{m}$ de espesor, posteriormente los cortes fueron procesados mediante técnicas histológicas de rutina $\mathrm{H}$ \& E lo cual permite observar la histología general de la aleta.

Para evaluar la expresión de la proteína Sonic hedgehog (SHH), se utilizaron los mismos 30 alevines del análisis histológico, se realizó una técnica inmunohistoquímica con el anticuerpo anti Shh (Shh, H-160, Santa Cruz). Desde los alevines incluidos en paraplast, se obtuvieron cortes de $5 \mu \mathrm{m}$, con micrótomo Microm (HM315R) los cuales se adhirieron a portaobjetos con carga positiva (Citoglas). Se adhirieron 5 cortes por portaobjeto, considerando un total de 2 portaobjetos por alevín. La recuperación de antígenos fue realizada en vaporera por 40 minutos, con los cortes sumergidos en Antigen unmasking solution (Vector). Se realizó bloqueo de peroxidasa endógena con peróxido de hidrógeno en metanol y bloquéo inespecífico de proteínas con PBS+BSA al $3 \%$. Para la incubación con el anticuerpo primario Shh (Shh, H-160, Santa Cruz) se usó una dilución 1:100 en buffer fosfato salino (PBS). Para la detección del anticuerpo primario se incubó con polímero conjugado con anticuerpo anti conejo y peroxidasa de rábano (HRP) (Zymed) durante $15 \mathrm{~min}$. Como sustrato para la HRP se usó diaminobencidina (DAB, Vector Labs). El control negativo estuvo dado por el desarrollo de la técnica inmunohistoquímica completa, pero sin considerar los anticuerpos primarios. Como control positivo interno se consideró la notocorda. Los cortes sagitales seriados de los peces fueron analizados consignando los tejidos que fueron marcados positivamente.

Análisis estadístico. A los valores obtenidos para las variables cuantitativas peso y longitud de cuerpo, largo y ancho de aleta caudal, se les realizó estadística descriptiva y fueron sometidos a prueba de normalidad de Shapiro-Wilk. Las diferencias observadas entre peces normales y deformes, fueron analizadas mediante prueba t de Student o U de Mann Whitney, utilizando el paquete estadístico IBM SPSS 20.0 


\section{RESULTADOS}

Cuando se compararon, las tres unidades de cultivo (lote) de la piscicultura se encontraron alevines deformes en las tres, pero hubo diferencias en la frecuencia y porcentaje de los peces severamente deformados, estando mucho más afectada la línea 1 de incubación con un 18,6\% en comparación con las otras dos líneas que presentaron solo $9,8 \%$ y $9,5 \%$ (Tabla I).
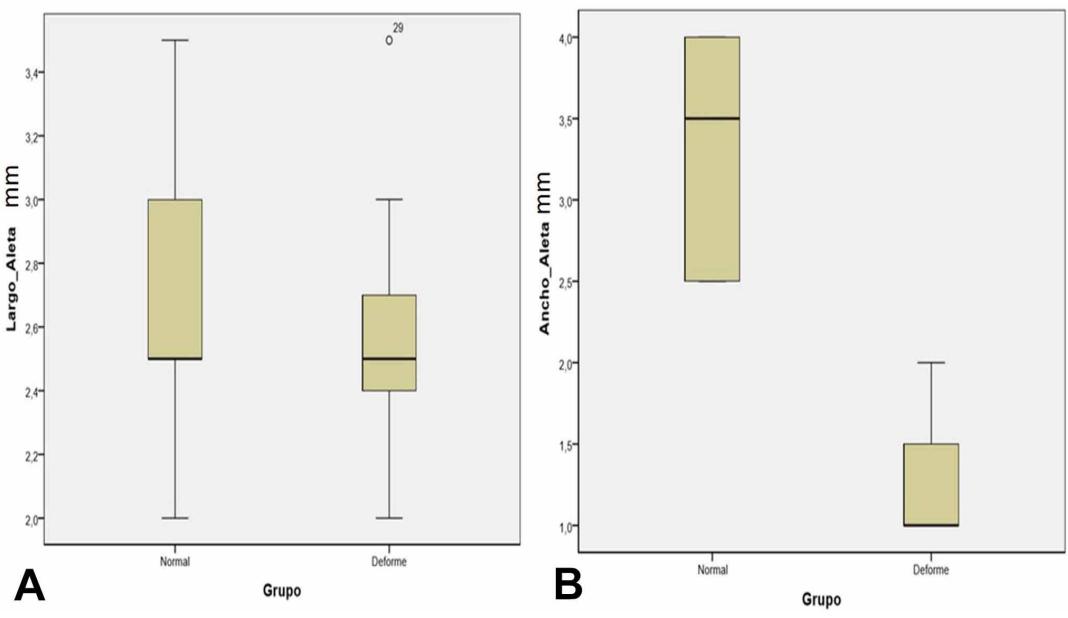

Fig.1) Diagrama de cajas de la longitud de la aleta caudal en milímetros (mm) en alevines de Salmón del Atlántico (Salmo salar) normales y deformes. Fig. 2) Diagrama de cajas del ancho de la aleta caudal de alevines de Salmón del Atlántico (Salmo salar) normales y deformes.
El peso de los alevines normales fue similar al de peso de alevines deformes ( $p>0,05)$, al igual que la longitud de la aleta caudal $(\mathrm{p}>0,05)$ (Fig. 1) y el ancho de la aleta de los alevines deformes fue inferior (mediana $1 \mathrm{~mm}$ ) al de los alevines normales (mediana 3,5 mm) $(\mathrm{p}<0,05)$ (Fig. 2, Tabla II).

Todos los análisis de laboratorio realizados para evaluar el estado sanitario de los peces resultaron negativos, indicando que los peces no presentaban patógenos virales ni tampoco presentaban patógenos bacterianos.

En los alevines eclosionados (475 UTAs) no se observaron cambios morfológicos, pero a las 600 UTAs se visualizó una deformación en la aleta caudal, la cual evolucionó en frecuencia y severidad hasta los 700 UTA (Tabla I, Figs 3A, alevín normal, 3B, aleta moderadamente deformada), 3C y 3D aleta severamente deformada).

Tabla I. Frecuencia de deformación y severidad de los hallazgos morfológicos observados en la aleta caudal de unidades de cultivo. de Salmon del Atlántico (Salmo salar) analizados.

\begin{tabular}{ccccccc}
\hline Unidad de cultivo-lote & \multicolumn{2}{c}{ Normal } & \multicolumn{2}{c}{$\begin{array}{c}\text { Deformación aleta caudal leve a } \\
\text { moderado }\end{array}$} & \multicolumn{2}{c}{$\begin{array}{c}\text { Deformación aleta caudal } \\
\text { Severo }\end{array}$} \\
\cline { 2 - 7 } & Frecuencia & Porc entaje & Frecuencia & Porcentaje & Frecuencia & Porcentaje \\
\hline Línea 1 Lote 3 & $231 / 334$ & $69,1 \%$ & $41 / 334$ & $12,3 \%$ & $62 / 334$ & $18,6 \%$ \\
Línea 3 Lote 4 & $200 / 256$ & $78,1 \%$ & $31 / 256$ & $12,1 \%$ & $25 / 256$ & $9,8 \%$ \\
Línea 5 Lote 6 & $258 / 316$ & $81,6 \%$ & $28 / 316$ & $8,9 \%$ & $30 / 316$ & $9,5 \%$ \\
\hline
\end{tabular}

Tabla II. Descripción estadística de las variables peso (g) y longitud del cuerpo (mm) y largo y ancho de la aleta $(\mathrm{mm})$ en alevines de Salmón del Atlántico (Salmo salar) normales y deformes.

\begin{tabular}{clcccccc}
\hline Variable & Grupo & N & Mín & Máx & Promedio & DE & SEM \\
\hline \multirow{2}{*}{ Peso de cuerpo } & Normal & 15 & 7,0 & 18 & 13,2 & 3,4 & 0,87 \\
& Deforme & 15 & 9,0 & 16 & 11,4 & 1,6 & 0,42 \\
\multirow{3}{*}{ Long de cuerpo } & Normal & 15 & 18 & 22 & 19,3 & 1,2 & 0,30 \\
& Deforme & 15 & 17 & 22 & 19,7 & 1,5 & 0,39 \\
& Lormal & 15 & 2,0 & 3,5 & 2,6 & 0,4 & 0,11 \\
\multirow{3}{*}{ Ancho de aleta ale ta } & Deforme & 15 & 2,0 & 3,5 & 2,5 & 0,4 & 0,11 \\
& Normal & 15 & 2,5 & 4,0 & $3,3 \mathrm{a}$ & 0,7 & 0,18 \\
& Deforme & 15 & 1,0 & 2,0 & $1,2 \mathrm{~b}$ & 0,3 & 0,81 \\
\hline
\end{tabular}

$\mathrm{a}, \mathrm{b}$ : Letras diferentes indican diferencias estadísticamente significativas $(\mathrm{p}<0,05)$ 

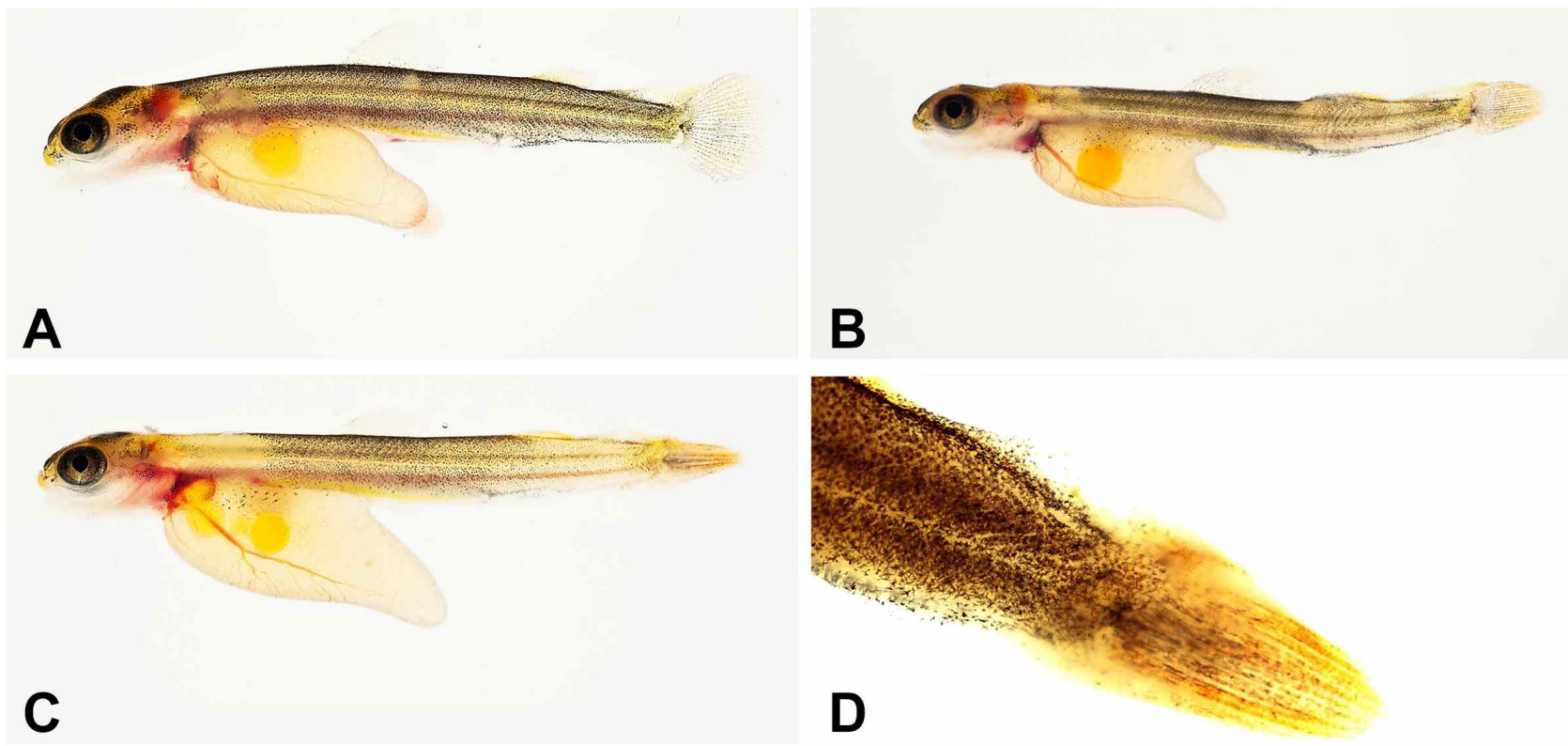

Fig. 3. Diferentes estados de deformación de la aleta caudal 3A. Nnormal; 3B. Deformación moderada; 3C. deformación severa y 3D cuadro de deformación severa de la aleta caudal, con fusión de lepidotriquias.

En las aletas caudales de los alevines normales, los rayos dérmicos llamados Lepidotriquias presentan gran cantidad de glicosaminoglicanos y se pueden visualizar mediante el azul de Alcián (Fig.s 4 y 5). Cada Lepidotriquia presenta segmentos cortos separados por articulaciones y en la parte más distal terminan en Actinotriquias (Fig.s 4 y 5). Las Lepidotriquias están revestidas por escleroblastos.

Después de las 600 UTAs de incubación, se evidenciaron los primeros cambios morfológicos: a) ausencia de surco entre los lóbulos dorsal y ventral b) las Actinotriquias se pliegan y adhieren entre ellas (Fig. 6), c) desorganización de las Lepidotriquias, ondulación y fractura de ellos (Figs. 3B y 7), d) se pierde la forma de abanico y adquiere la forma aguzada (Figs. 3C, 3D y 8), presentando una base de $3 \mathrm{~mm}$ que se reduce a $1+0.5 \mathrm{~mm}$ en el extremo distal. Se pierde totalmente el ordenamiento espacial de rayos e interrayos y la distancia entre ellos (Figs. 3C, 3D, 8).

En los alevines deformes también se encontraron las Lepidotriquias (revestidos por escleroblastos (son comparables a células osteógenas) de los cuáles podemos ver sus núcleos aplanados (Figs. 9 y 10). En las zonas interrayos observamos vasos sanguíneos de distinto calibre ocupando la mayor parte de ella (Figs. 9 y 10). Además soluciones de continuidad en el endotelio (ura 11) y zonas hemorrágicas (eritrocitos, linfocitos, núcleos). El morfógeno Shh resultó positivo en núcleos de células basales de la epidermis, y en escleroblastos de Lepidotriquias, como también en su control positivo que era la notocorda (Figs. 12 y 13).

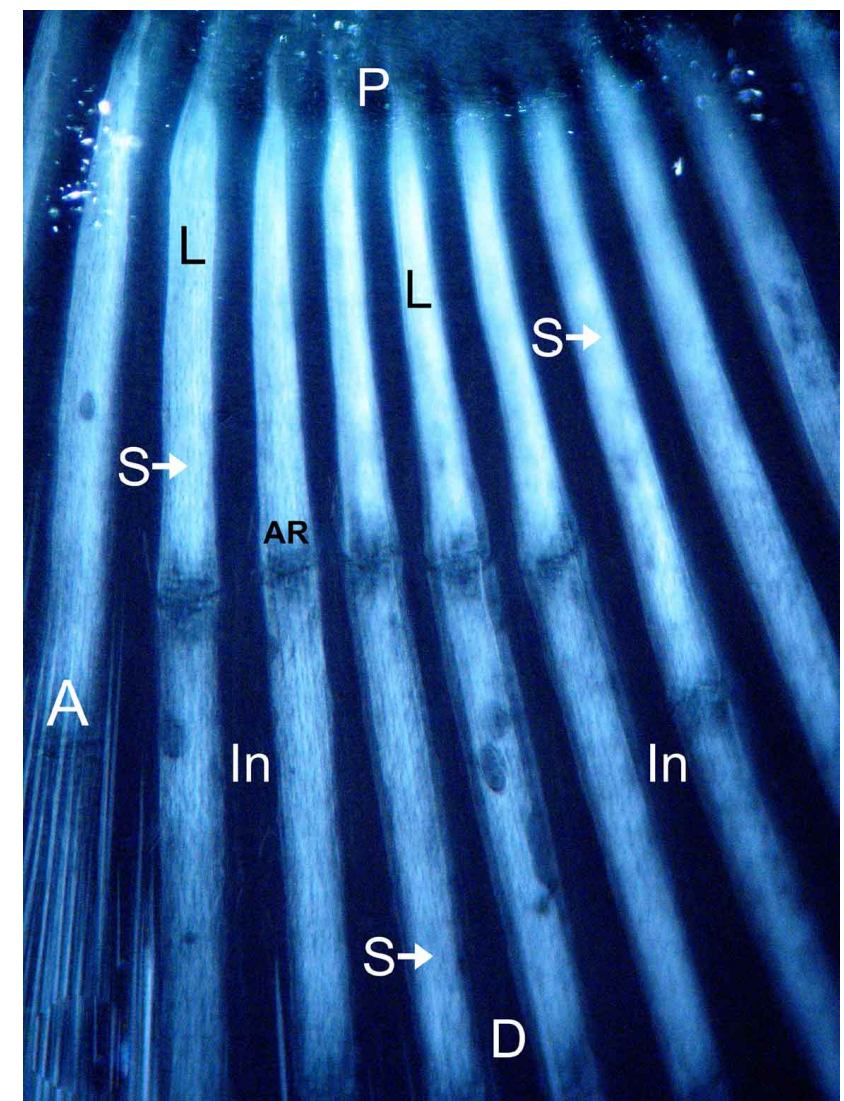

Fig. 4. Aleta de alevín de 760 UTAs normal. Los rayos o lepidotriquias (L), están mas separados en la región distal (D) que en la proximal (P), Interrayos (In) segmentos (s). articulaciones (AR). Actinotriquias (A). Técnica de Hanken y Wassersug, y microscopía de polarización $100 \mathrm{X}$. 


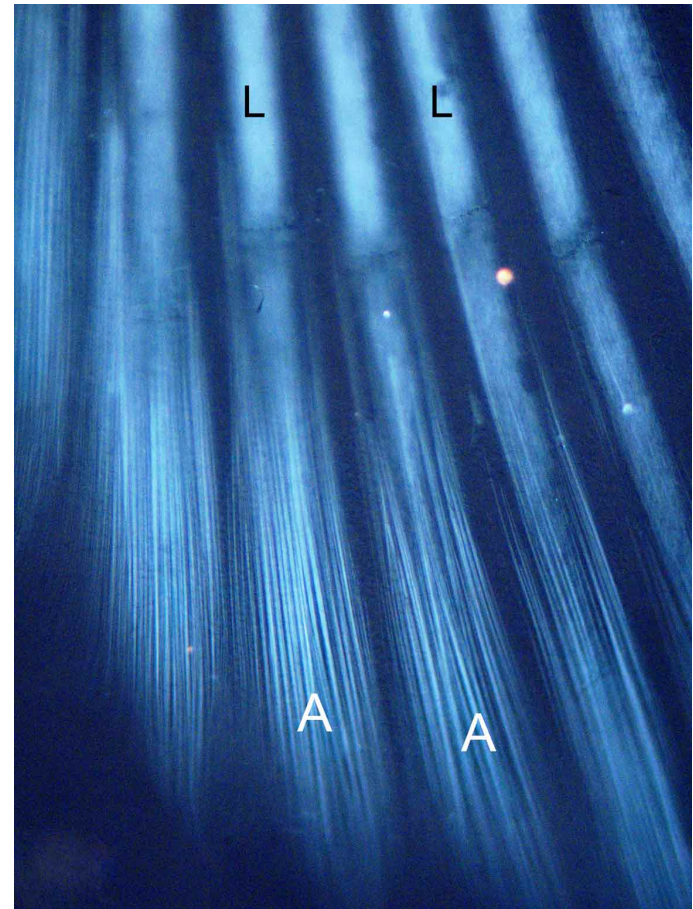

Fig. 5. Aleta de alevín normal de 760UTAs. Actinotriquias (A), Lepidotriquias (L). Técnica de Hanken y Wassersug, y microscopía de polarización 100X.

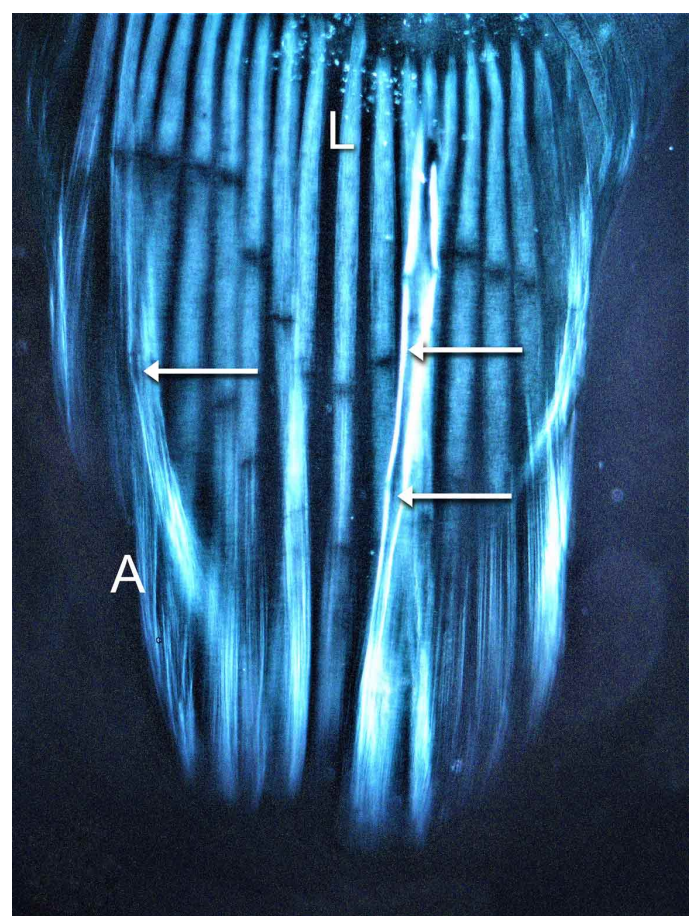

Fig. 7. Aleta moderadamente deformada de alevin de 760 UTAs. Algunas Lepidotriquias están dobladas y fracturadas (ver flechas). Las Lepidotriquias(L) no son homogéneas y se juntan en la región más distal, disminuyendo los segmentos interrayos. Técnica de Hanken y Wassersug, y microscopía de polarización 100X.

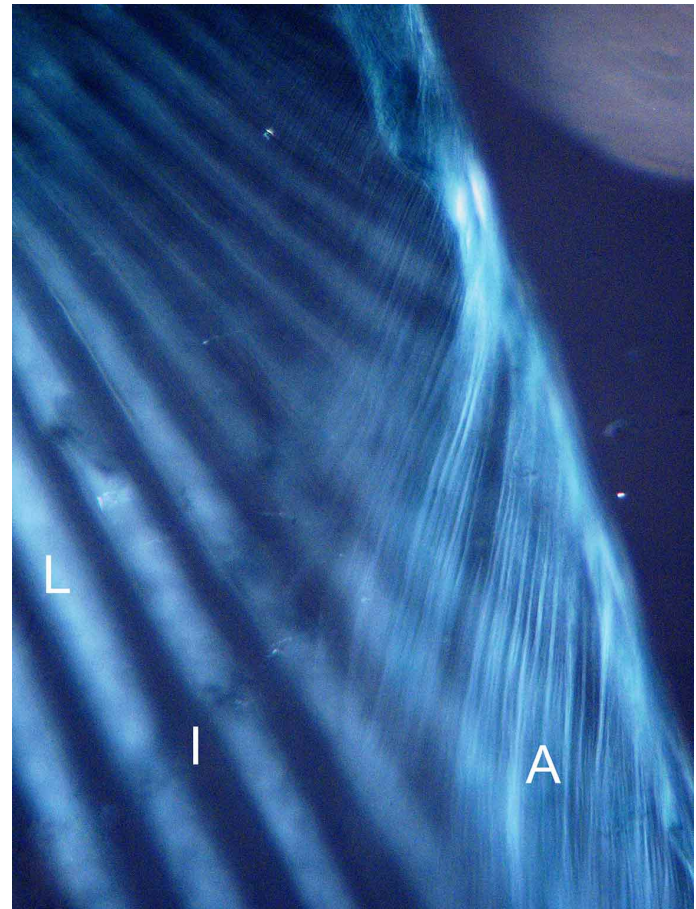

Fig. 6. Aleta con deformación inicial de alevin de 760 UTAs. La Actinotriquias (A) más periféricas se doblan. Técnica de Hanken y Wassersug y microscopía de polarización 100X.

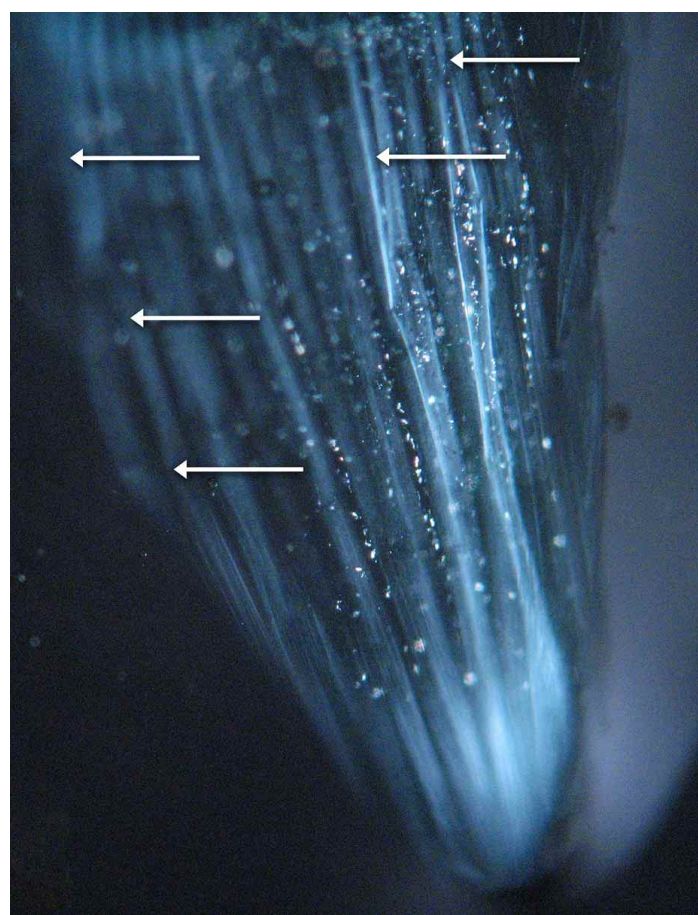

Fig. 8. Es una aleta aguzada de alevín de 760 UTAs. Las lepidotriquias son muy delgadas, débiles, se fracturan (ver flechas) y se adhieren unas a otras en la parte mas distal de la aleta. Técnica de Hanken y Wassersug, y microscopía de polarización $100 \mathrm{X}$. 


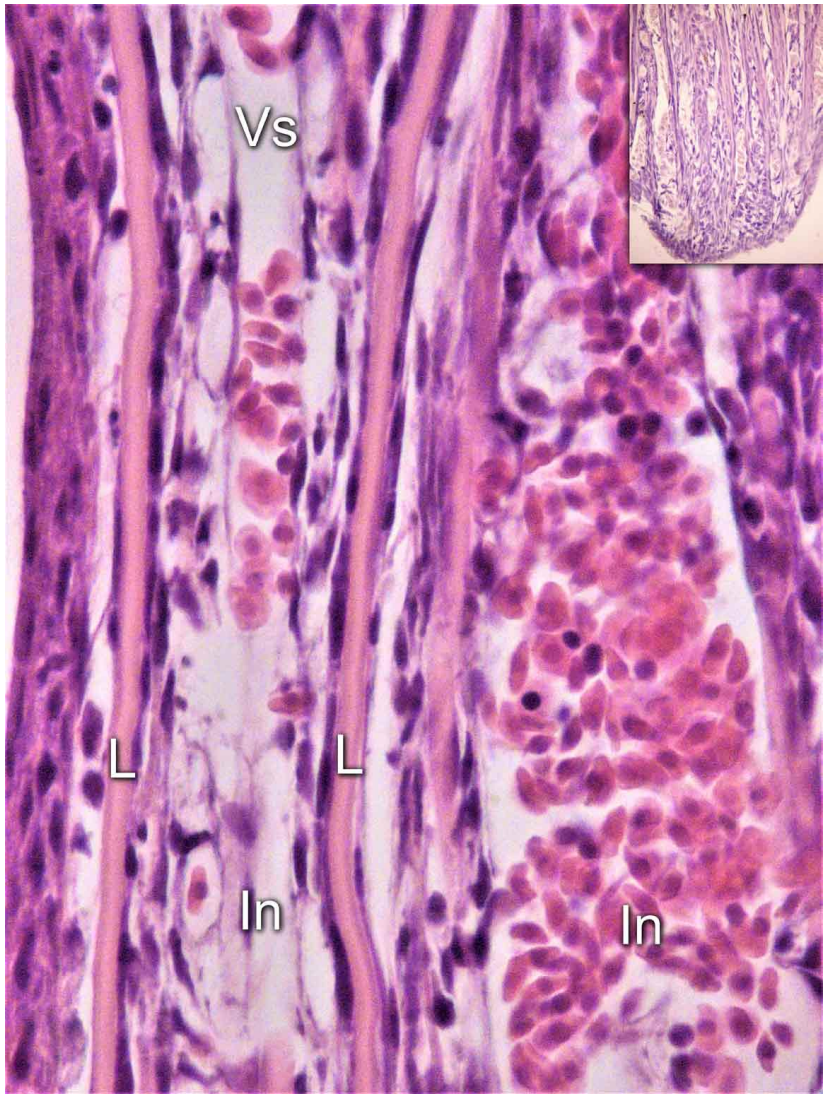

Fig. 9. Corte histológico de la región más distal de la aleta caudal deforme de alevín de 700 UTAs. Se observan Lepidotriquias (L), Zonas interrayos (In) con vasos sanguíneos (Vs). 400X, En el recuadro se ve una visión panorámica de la aleta. H-E azul de Alcián.

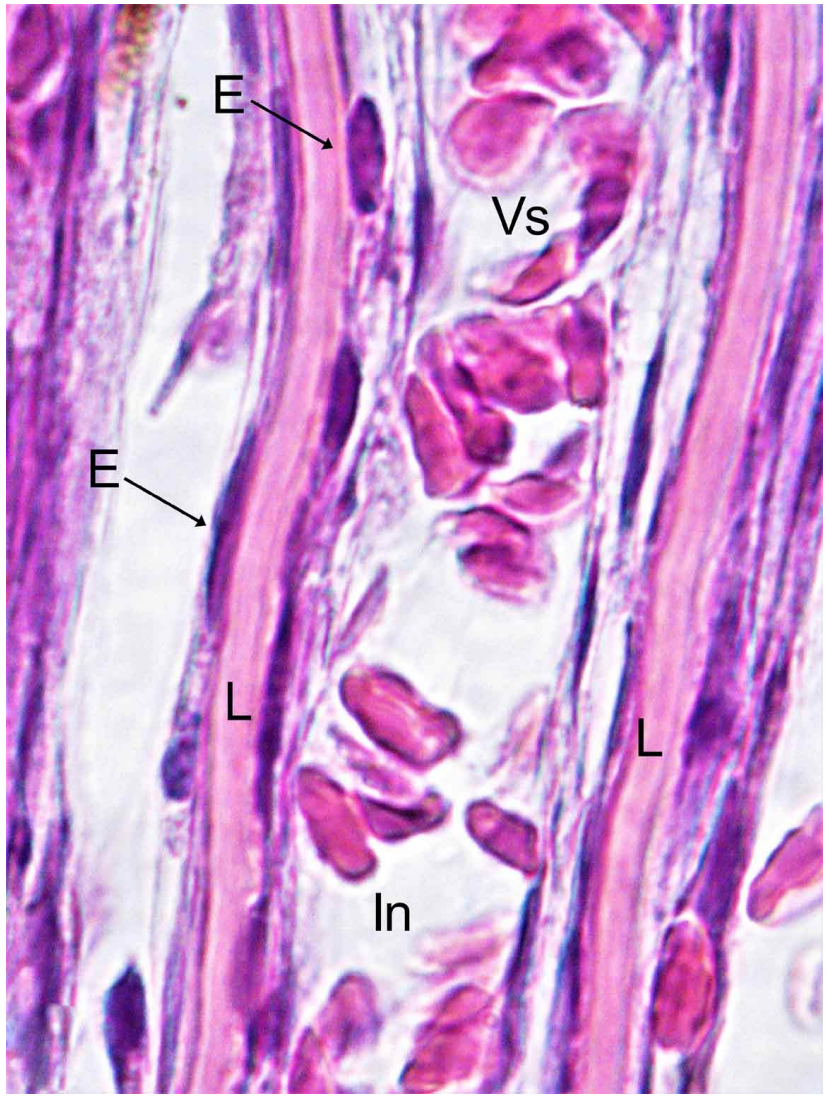

Fig. 10. Corte histológico de la región más distal de la aleta caudal deforme de alevín de 700 UTAs. Escleroblastos (E) en las lepidotriquias (L) y vasos sanguíneos (Vs) en los espacios interrayos (In). 1000X H-E azul de Alcián.

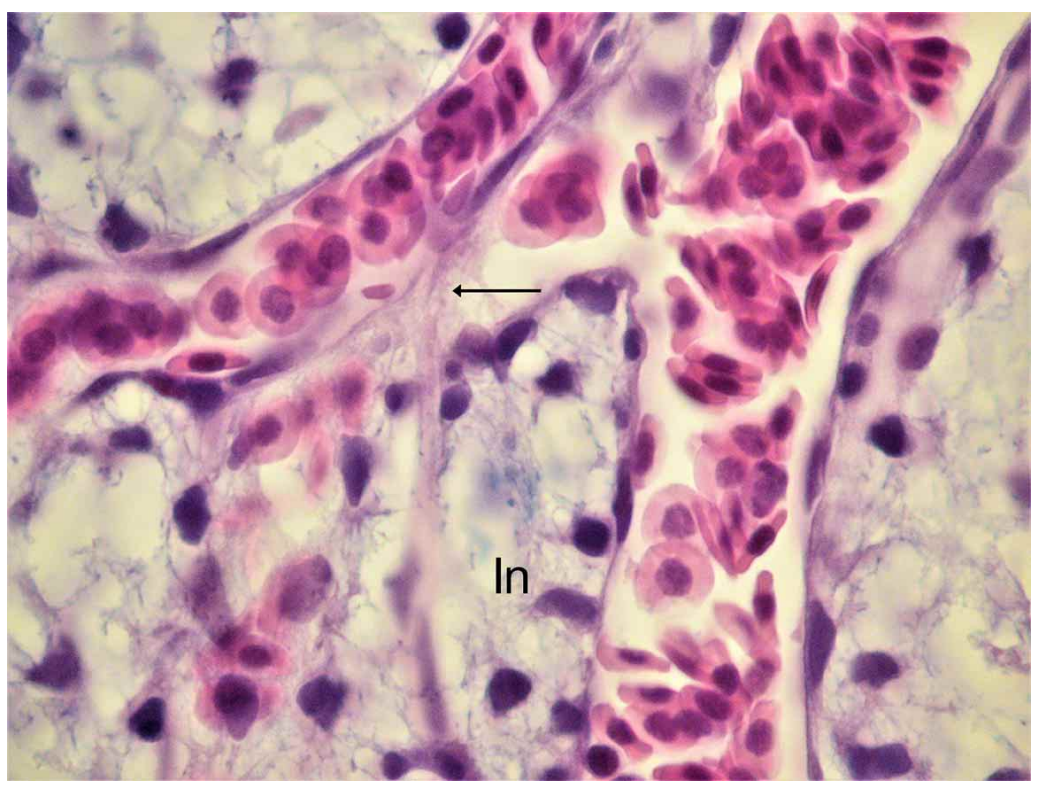

Fig. 11. Corte histológico de la aleta caudal deformada. En las zonas inter-rayos (In) Los vasos sanguíneos presentan solución de continuidad del endotelio (ver flecha) $1000 \mathrm{X}$.

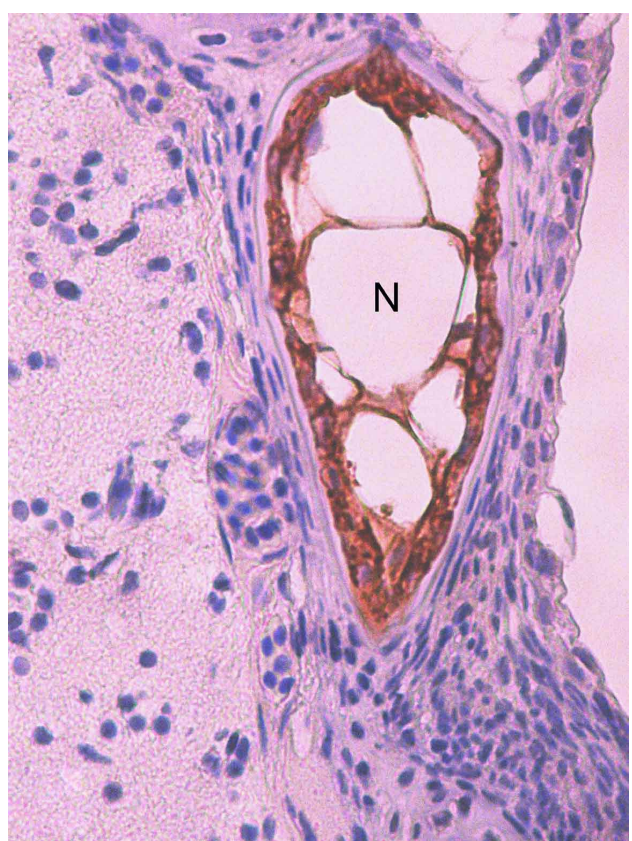

Fig. 12. Notocorda (N)de alevín con deformación de la aleta caudal. Control positivo para Shh. 200X 


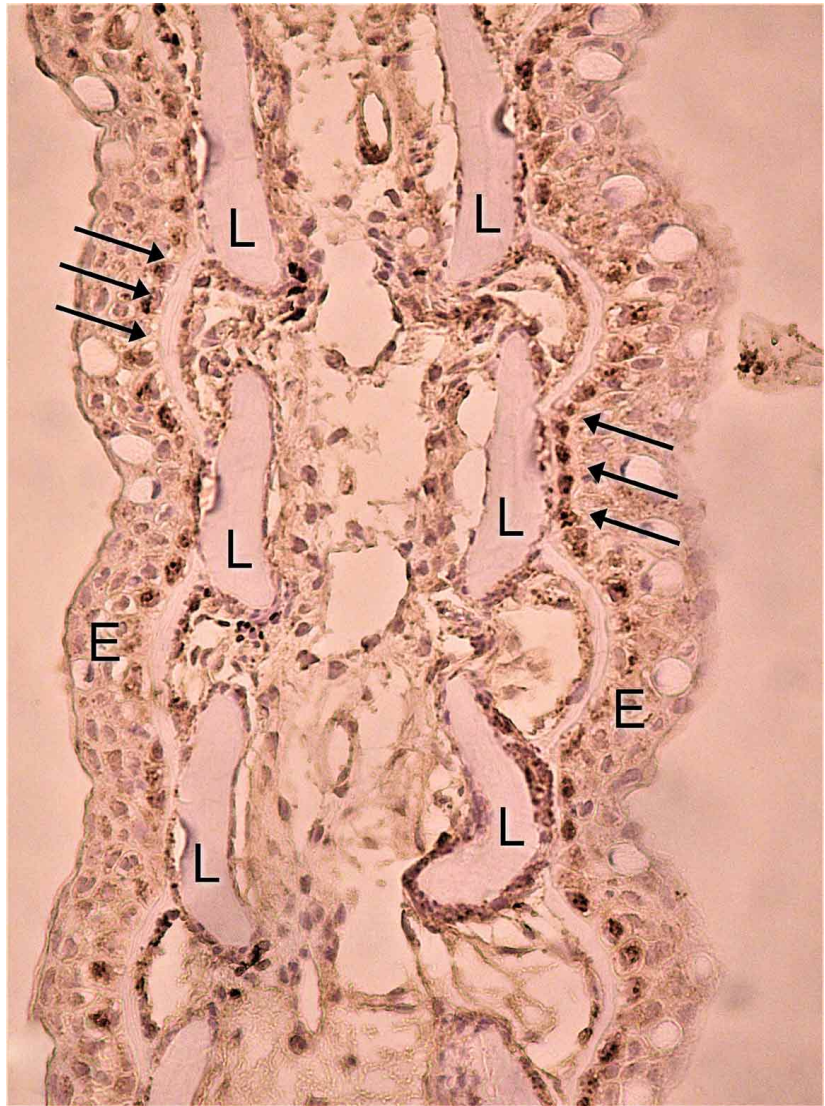

Fig. 13. Corte transversal de aleta caudal de alevín deforme de 700 UTAs. Se observa Epitelio de revestimiento (E) Lepidotriquias (L). Los núcleos de la capa basal del epitelio de revestimiento (ver flechas) y de los escleroblastos son inmunopositivos (ver flechas). 200X

\section{DISCUSIÓN}

En las tres unidades de cultivo de la piscicultura se encontró mas de un $80 \%$ de los alevines normales y menos de un $20 \%$ estaban deformes. El hecho de que los alevines presentaron una aleta caudal de menor ancho tomando una forma aguzada, impide la normal natación. En los peces adultos la aleta se puede regenerar completamente (MaríBeffa et al., 1999; Rolland-Lagan et al., 2012), sin embargo, en los alevines severamente deformados, esto no ocurrió, no obstante, estos mostraron señales que indicaban la presencia de centros emisores de morfógenos activos. Algunos autores como Nakatani et al. (2008) han explicado que se tiene que gatillar la migración de células mesenquimales desde el blastema para que pueda ocurrir la regeneración de la aleta caudal. En los alevines este proceso no ocurrió debido a las alteraciones encontradas en los vasos sanguíneos, del blastema como soluciones de continuidad, hemorragias y bacterias.
La formación de las Lepidotriquias se inicia por el contacto de la epidermis con el tejido mesenquimático subyacente. Según Akimenko et al. (2003) la epidermis tiene un papel fundamental en el proceso de formación de Lepidotriquias. En este estudio se pudo observar la presencia del morfógeno Shh en la capa basal de la epidermis y también en los escleroblastos de las Lepidotriquias y endotelio de algunos vasos sanguíneos. Esto se relaciona con los estudios de Wargelius et al. (2009) y Rojas et al. (2016) que sugieren que Shh puede ser usado como un marcador de proliferación de osteoblastos o escleroblastos. Otros autores, como Laforest et al. (1998) y Rojas et al. (2014), indican la importancia de los genes Shh en la formación de los patrones de las Lepidotriquias, existiendo una gradiente de señalización de Shh que regula la expresión diferencial de genes de una manera dependiente de la concentración. Este morfógeno siempre produce inmunomarcación en la notocorda del salmón, por lo tanto, se utiliza esta estructura como control positivo (Rojas et al., 2015).

Existen varios factores ambientales que pueden causar deformidades de la aleta caudal, incluidos los efectos producidos por altas temperaturas, la contaminación por metales pesados, los efectos de la polución (Jawad et al., 2018), o los efectos producidos por luz continua en el desarrollo óseo (Wargelius et al.). Nosotros descartamos los factores sanitarios debido a que los peces resultaron negativos a patógenos virales y bacterianos. En este caso, la causa fue conocida y estaba relacionado con cambios bruscos de la temperatura del agua y aunque no fue alterado el nivel de la expresión de los morfógenos Shh desde el epitelio, hubo cambios histológicos en el blastema interrayos lo cual afectó la formación de las Lepidotriquias. Descartamos a la hipoxia como factor etiológico, porque en hipoxia aumentan los brotes angiogénicos y el número de vasos sanguíneos, lo cual en este caso no ocurre y además porque la hipoxia en alevines se relaciona con deformidad de la columna vertebral (Castro Sánchez et al., 2011) y no por deformidad de aletas. Una vez establecidas las condiciones de cultivo la condición descrita disminuye significativamente hasta llegar a cero.

CONCLUSIONES. La deformación se observa desde los 600 UTAs, Lepidotriquias de la aleta caudal se desorganizan, fracturan y no se regeneran. Existe también una vasodilatación y solución de continuidad de endotelio y hemorragias en las zonas interrayos. Los centros emisores de señales para la regeneración de Lepidotriquias permanecieron activos en la epidermis pero el blastema subyacente estaba alterado en los peces que enfermaron. Este defecto se gatillaría por errores del manejo perieclosional, como cambios bruscos de la temperatura. 
AGRADECIMIENTOS. Para Soraya Gallegos Riffo. Jefa del Centro de Incubación de la Piscícola que facilitó que este estudio se llevara a cabo.

ROJAS, M.; PELLÓN, M.; CERDA, C.; ULLOA, R.; SUAREZ, R.; GODOY, M. \& DEL SOL, M. Deformation of the caudal fin in Atlantic salmon fry (Salmo salar), in the yolk sac stage. Int. J. Morphol., 38(6):1631-1638, 2020.

SUMMARY: This study analyzes the deformity of Atlantic salmon fry (Salmo salar) caudal fin, which gives it a "pointed tail" appearance. Although at hatching specimens were normal, the deformities were detected later. The objective of this work is to describe the anatomical and histological changes of the deformed caudal fin of the Atlantic salmon fry (Salmo salar). In this analysis we used 60 specimens for laboratory analyses, to rule out the presence of viral or bacterial pathogens. Another 60 fry, developmental stage between 600 and 700 Accumulated Thermal Units (UTAs) were anesthetized with $5 \%$ Benzocaine, fixed in $10 \%$ formalin, weighed and measured. Of these, a group of 30 fry ( 15 normal and 15 deformed) underwent the Hanken and Wassersug technique to evaluate lepidotrychia or dermal rays. The remaining 30 fry (15 normal and 15 deformed) were processed using $\mathrm{H} \& \mathrm{E} /$ Alcián blue histochemical techniques to evaluate general histological characteristics. Additionally, immunohistochemical techniques were used to determine the location and presence of Sonic hedgehog (Shh) signal centers for lepidotrychia development. The values obtained for the quantitative variables body weight, length and width of the tail fin were described using the arithmetic mean and standard deviation. The deformity was observed from 600 UTA. Weight of deformed fry was less than normal fish, length of the fin was similar in normal and deformed fish. In contrast, width of the deformed fin was significantly reduced. The sharp fin phenotype presented a change in the conformity of the edges, lepidotrychia fractures. The epidermis presented active Shh signaling centers, but the interray blastema showed vasodilation, congestion and hemorrhages. The presentation of this case was related to sudden increases in perieclosional temperature.

KEY WORDS: Atlantic salmon, Salmo salar; Caudal fin; Deformity.

\section{REFERENCIAS BIBLIOGRAFICAS}

Akimenko, M. A.; Marí-Beffa, M.; Becerra, J. \& Géraudie, J. Old questions, new tools, and some answers to the mystery of fin regeneration. Dev. Dyn., 226(2):190-201, 2003.

Anacker, R. L. \& Ordal, E. J. Studies on the myxobacterium Chondrococcus columnaris. I. Serological typing. J. Bacteriol., 78(1):25-32, 1959.

Blake, S. L.; Schill, W. B.; McAllister, P. E.; Lee, M. K.; Singer, J. T. \& Nicholson, B. L. Detection and identification of aquatic birnaviruses by PCR assay. $J$. Clin. Microbiol., 33(4):835-9, 1995.

Castro Sánchez, R.; Bustos Obregón, E. \& Rojas Rauco, M. Hypoxia is like an ethiological factor in vertebral column deformity of salmon (Salmo salar). Aquaculture, 316(1-4):13-9, 2011.

Chase, D. M.; Elliott, D. G. \& Pascho, R. J. Detection and quantification of Renibacterium salmoninarum DNA in salmonid tissues by real-time quantitative polymerase chain reaction analysis. J. Vet. Diagn. Invest., 18(4):375-80, 2006.
Jawad, L. A.; Ibrahim, M. \& Waryani, B. Incidences of caudal fin malformation in fishes from Jubail City, Saudi Arabia, Arabian Gulf. Fish. Aquat. Life, 26(1):65-71, 2018

Laforest, L.; Brown, C. W.; Poleo, G.; Géraudie, J.; Tada, M.; Ekker, M. \& Akimenko, M. A. Involvement of the sonic hedgehog, patched 1 and bmp2 genes in patterning of the zebrafish dermal fin rays. Development, 125(21):4175-84, 1998

Marí-Beffa, M.; Palmqvist, P.; Marín-Girón, F.; Montes, G. S. \& Becerra, J. Morphometric study of the regeneration of individual rays in teleost tail fins. J. Anat., 195(Pt. 3):393-405, 1999.

Nakatani, Y.; Nishidate, M.; Fujita, M.; Kawakami, A. \& Kudo, A. Migration of mesenchymal cell fated to blastema is necessary for fish fin regeneration. Dev. Growth Differ., 50(2):71-83, 2008

Olsen, A. B.; Hjortaas, M.; Tengs, T.; Hellberg, H. \& Johansen, R. First description of a new disease in rainbow trout (Oncorhynchus mykiss (Walbaum)) similar to heart and skeletal muscle inflammation (HSMI) and detection of a gene sequence related to piscine orthoreovirus $(P R V)$. PloS One, 10(7):e0131638, 2015.

Quint, E.; Smith, A.; Avaron, F.; Laforest, L.; Miles, J.; Gaffield, W \& Akimenko, M.A. Bone patterning is altered in the regenerating zebrafish caudal fin after ectopic expression of sonic hedgehog and bmp2b or exposure to cyclopamine. Proc. Natl. Acad. Sci. U. S. A., 99(13):8713-8, 2002.

Rojas, M.; Pellón, M. \& del Sol, M. Salmon caudal fin development (Salmo salar). Int. J. Morphol., 33(2):514-21, 2015.

Rojas, M.; Saint-Pierre, G.; Hartley, R.; Vásquez, B.; Conei, D. \& del Sol., M. Immunolocalization of morphogen sonic hedgehog in salmon fry (Salmo salar). Int. J. Morphol., 34(2):770-4, 2016.

Rojas, M.; Signore, I. A. \& Mejías, R. Morphogens during embryonic development of vertebrates. Int. J. Morphol., 32(1):319-26, 2014.

Rolland-Lagan, A. G.; Paquette, M.; Tweedle, V. \& Akimenko, M. A. Morphogenbased simulation model of ray growth and joint patterning during fin development and regeneration. Development, 139(6):1188-97, 2012.

Snow, M.; McKay, P.; McBeath, A. J. A.; Black, J.; Doig, F.; Kerr, R.; Cunningham, C. O.; Nylund, A. \& Devold, M. Development, application and validation of a Taqman real-time RT-PCR assay for the detection of infectious salmon anaemia virus (ISAV) in Atlantic salmon (Salmo salar). Dev. Biol. (Basel), 126:133-45, 2006

Takano, T.: Nawata, A.; Sakai, T.; Matsuyama, T.; Ito, T.; Kurita, J.; Terashima, S.; Yasuike, M.; Nakamura, Y.; Fujiwara, A.; et al. Full-genome sequencing and confirmation of the causative agent of erythrocytic inclusion body syndrome in coho salmon identifies a new type of piscine orthoreovirus. PLoS One, 11(10): $0165424,2016$.

Wargelius, A.; Fjelldal, P. G.; Nordgarden, U. \& Hansen, T. Continuous light affects mineralization and delays osteoid incorporation in vertebral bone of Atlantic salmon (Salmo salar L.). J. Exp. Biol., 212(Pt. 5):656-61, 2009.

Whitman, K. A. Finfish and Shellfish Bacteriology Manual: Techniques and Procedures. Ames, Iowa State Press. 2004.

Wiklund, T.; Madsen, L.; Bruun, M. S. \& Dalsgaard, I. Detection of Flavobacterium psychrophilum from fish tissue and water samples by PCR amplification. J. Appl. Microbiol., 88(2):299-307, 2000.

Dirección para correspondencia:

Dra. Mariana Rojas R.

Laboratorio de Embriología Comparada

Programa de Anatomía y Biología del Desarrollo. ICBM

Facultad de Medicina

Universidad de Chile

CHILE

E-mail: dramrojas@hotmail.com

Recibido : 02-06-2020

Aceptado: 12-07-2020 